\title{
Design of Air Traffic Control Operation System Using Multi Agent Technology and Simulation
}

\author{
Zhipeng Wei ${ }^{*}$ and Jing Li
}

Kaifeng University, College of Information Engineering, Kaifeng, Henan, 475000, China

\begin{abstract}
Air traffic system is analyzed from the aspects of system's attributes internal operating mechanisms and external behaviors. A hybrid air traffic simulation model described by the combination of discrete event and continuoustime models is proposed. The simulation model uses Agent-based techniques to describe micro behaviors of the air traffic individuals and integrates micro behaviors into dynamic macro performance in system-wide level. Typical Agent-based air traffic models such as aircraft agent and controller agent have been constructed. An arrivals and departures simulation application has validated that the Agent based hybrid air traffic model is provided with high fidelity in micro behavior and macro performance of the system.
\end{abstract}

Keywords: Agent, air traffic simulation, continuous time, discrete event, macro performance, micro behavior.

\section{INTRODUCTION}

In recent years, the increasing air traffic demand, and the existing air traffic control support capabilities are limited, contradictory demand and supply have become increasingly prominent [1].

Whether adjust the existing transportation system elements airspace structure, route or routes, such as the introduction of new technologies and new concepts are needed in advance to make a security assessment to find potential risk factors. ATC system is a complex system; the use of computer simulation of air traffic control system is an important research tool. However, existing simulation tools have two problems, one, based on single centralized difficult to do large-scale simulation of microscopic simulation; Second, the lack of an analog controller simulation deployment capabilities. For these two questions, we studied the Distributed Artificial Intelligence-multi-Agent technology in air traffic control simulation; and used Java language to develop a national air traffic control system based on the field of the first prototype Multi-Agent regional control simulation [2].

Along with the continuous development of civil aviation, the large-scale construction of many airport facilities, the main airports are changing from the original single-airport terminal area to the multi-airport terminal area. In order to achieve the research of the actual capacity assessment of the complicated multi-airports terminal area, a more dynamic and complete simulating system of the multi-airports terminal area need to be created.

\section{RELATED TECHNOLOGIES}

A multi-Agent system (M.A.S.) is a computerized system composed of multiple interacting intelligent Agents within an environment. Multi-Agent systems can be used to solve problems that are difficult or impossible for an individual Agent or a monolithic system to solve. Intelligence may include some methodic, functional, procedural or algorithmic search,. find and processing approach (Fig. 1).

Air traffic operating model of multi-airport terminal area, which is based on the current question of terminal area capacity and the status of research on air traffic development, was discussed by applying distributed artificial intelligence Multi-Agent theories and methods, and the working mode of the multi-sect oral synergies between the aircraft, controllers, and the airport and so on was studied. The Multi-Agent System Simulation Mode is designed, and the specific design models of some Intelligent Agents, such as: Flight Agent [3], Controller Agent and Airport Control areas Agent were presented. As a whole framework and operational system of the multi-airport terminal area air traffic intelligent simulation system was made, which is based on the structures of the Multi-Agent. (Fig. 2) And finally, a foundation which is for reviewing the actual operation conditions of the multi-airport terminal area and the realization of the air traffic intelligent simulation system of the multi-airport terminal area has be laid.

The purpose of instrument flight procedure is to guarantee the safety and improve efficiency of air traffic operations in terminal area, and flight procedure design is a scientific planning and reasonable design work for the arrival and departure air routes and the network consisted of them.

Fig. (3) is one of the most important airspace resources, and the basis of airspace planning, air Flight procedure 


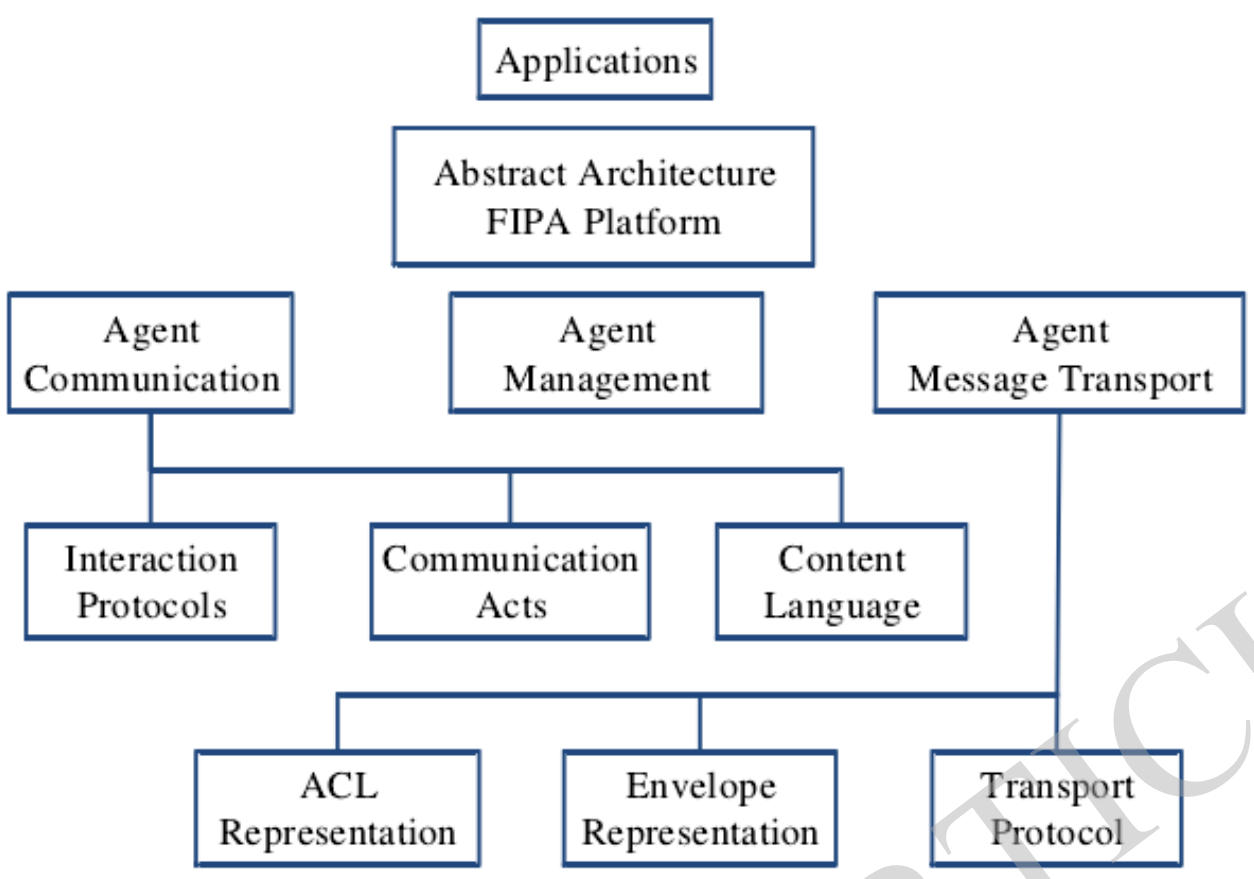

Fig. (1). Multi-agent system.

Fig. (2). JADE.

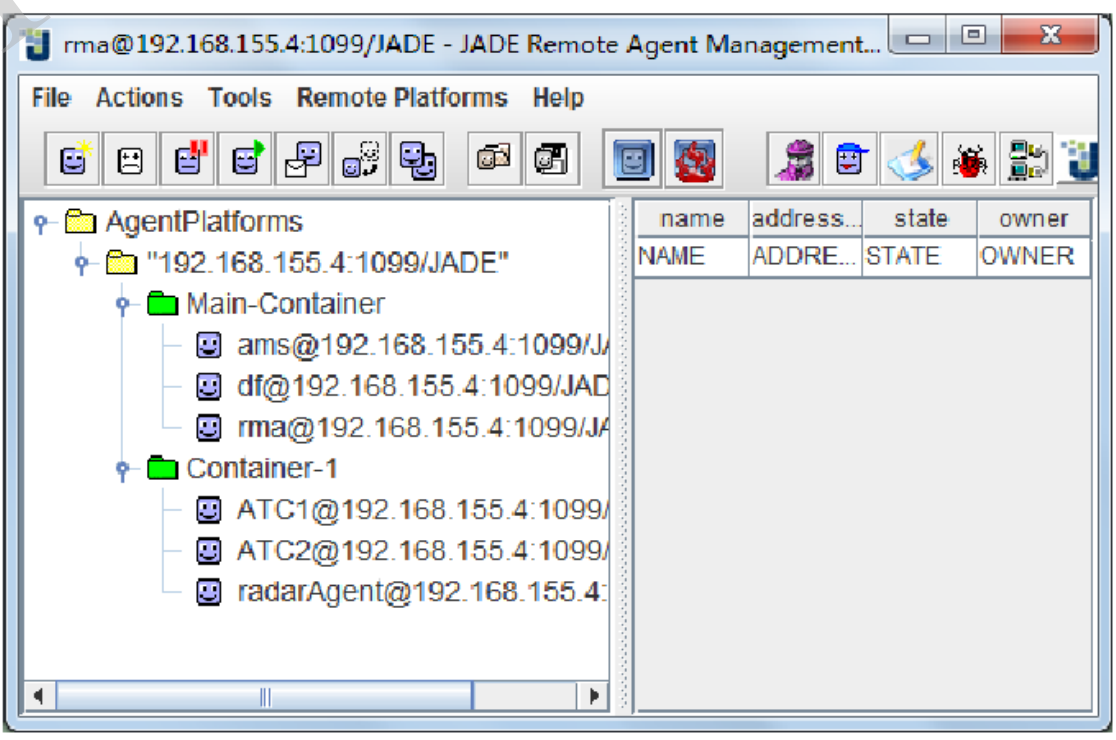

Fig. (3). JADE remote management GUI. 
traffic operation and airport constructions, etc. At the same time, procedure based operations become the direction of technology development in the future aviation transportation system. Evaluation of flight procedure's operation include works about audit, evaluation and certification for flight procedure's design schemes and it's implementations, and then evacuation is a necessary step to ensure the rationality, feasibility for flight procedure. However, there is still lack of integrated, effective theory, models and methods for flight procedure's assessment [4].

This thesis conducted in-depth analysis on the concept and connotation of flight procedure's evaluation problem, summarized the relevant aspects of the latest research achievements, advanced a new theoretical system in four aspects including safety risk, economic efficiency, air traffic serviceability and environment impact, and constructed a dynamic trajectory model with the combination of flight dynamics and kinetics, and a complete flight procedure's operation simulation and analysis software platform was developed based on the theory, models, and methods purposed above.

As shown in Figs. (4, 5), first of all, flight procedure's operation safety and risk were researched systematically from multiple sides, multiple stages and multiple levels. Safety and risk includes mainly the risk of proximity of terrains and air traffic conflict during flight. Proximity of terrains was consisting of two stages. The first stage includes departure procedures and the arrival, initial and inter-meditate segment of approach procedures. A cumulative risk model was established which calculated the probability of aircraft penetrating the obstacle clearance altitude. Aiming at the Instrument Landing System approach process, the total collision probability was analyzed among aircraft and buildings with different position relationship in the second stage [5].

Fig. (4). Physics and model.
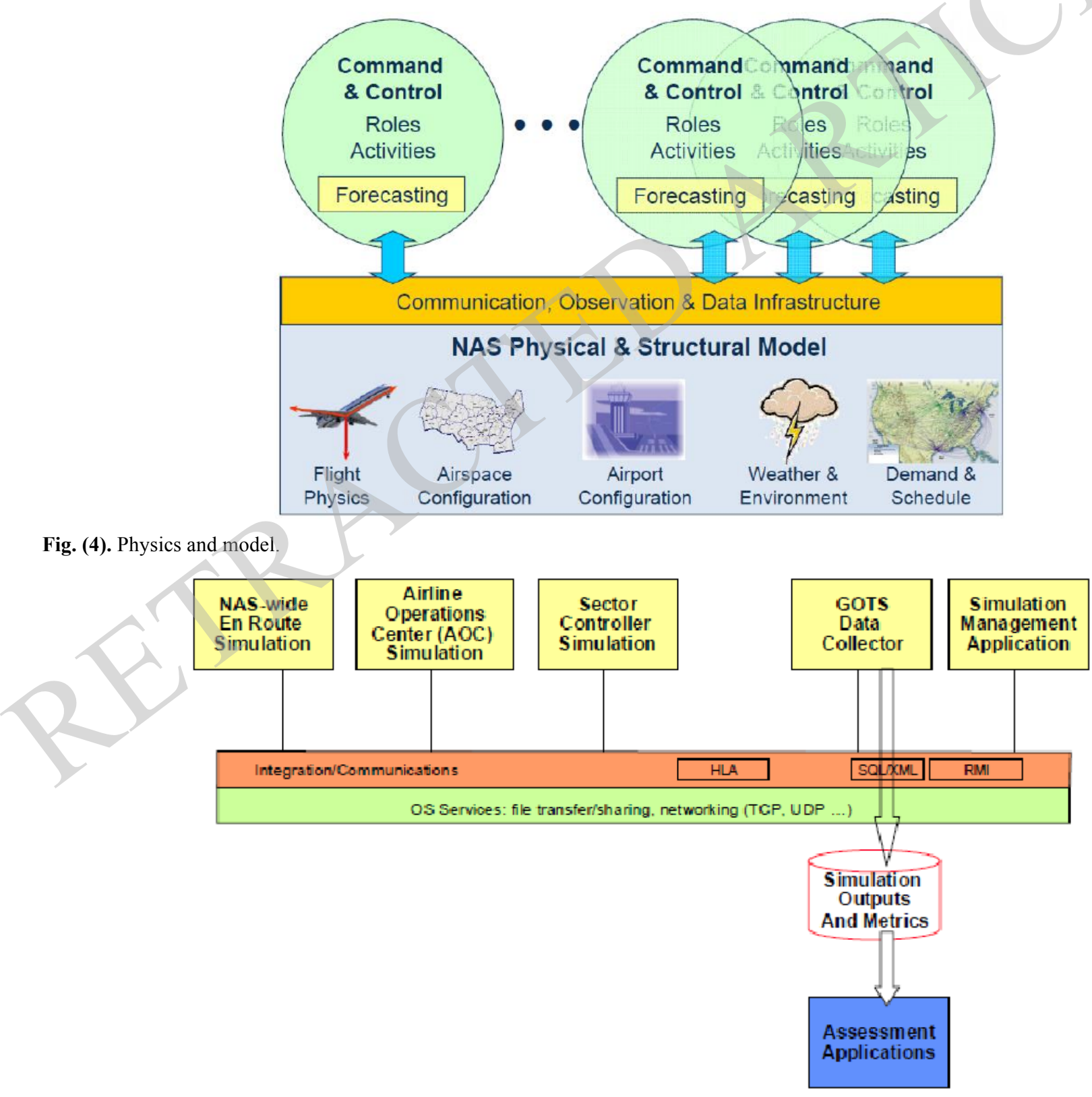

Fig. (5). ACES architecture. 
From macroscopic and microscopic levels, conflict risk phenomenon was researched respectively, aiming at the competition for spatiotemporal resources during multiple aircraft operations. From the perspective of prediction of the overall state of conflict, using the stochastic process method, the mathematical expectation and variance of conflict frequency were calculated among crossing or converging air traffic flows, then a macro conflict risk model was derived. [6]. A micro conflict risk model was analyzed which was oriented conflict evolution process, and three measure models about conflict severity, conflict intensity and cumulative risk were proposed. Finally, several case studies were designed and implemented, and the results of the experiments were analyzed (Fig. 6).

Economic analysis on flight procedure operation aimed at studying and revealing the potential space that enhanced economy by flight procedure's optimization. Combined with aircraft dynamic models, flight cost index and fuel consumption models, the concept and the calculation models of flight procedure operation cost were put forward innovative, and limited operation trajectory and reference operation trajectory were defined under specific airspace condition. According to a continuous descent approach or continuous climb departure mode, the vertical profile of a direct horizontal route which was defined by the start point and the end point of a flight procedure, was optimized using four orders Runge Kutta method, with the objective of minimize the overall flight cost. Then, the optimized trajectory was defined as a reference operation trajectory. Reference operation cost (ROC) and limited operation cost
(LOC) were calculated respectively according to the models mentioned in this chapter. At last, economic efficiency was proposed by a function of ROC and LOC, and case study was carried out [7].

\section{ANALYSIS OF INCLINATION}

In this thesis, air traffic control (ATC) serviceability of flight procedure is an entire innovative con-tent. Based on the characteristics analysis of history trajectory data of flight procedure a measurement method of trajectories similarity was established by the comparison of tracks which have the same time series. Trajectories data of flight procedures operation were clustered through hierarchical clustering method, and the mean flight trajectory was constructed according to each trajectory cluster. Then three ATC serviceability evaluation indicators and measurement methods were proposed, which include the concept and algorithms for longitudinal deviation lateral deviation and outlier's ratio. At last, a case study of trajectory clustering was implemented and the analysis result certificates the availability of clustering methods and the conceptual models of ATC serviceability. The environmental impacts resulted by flight procedure commonly operation include noise and exhaust gas emissions. Several noise impact evaluation models used and their limit standards were analyzed. Considering the shortages of airport noise assessment index system in China, a evaluation index and its limit value which is applicable to noise impact analysis of flight procedure operations, was pro-posed. A segmented trajectory model

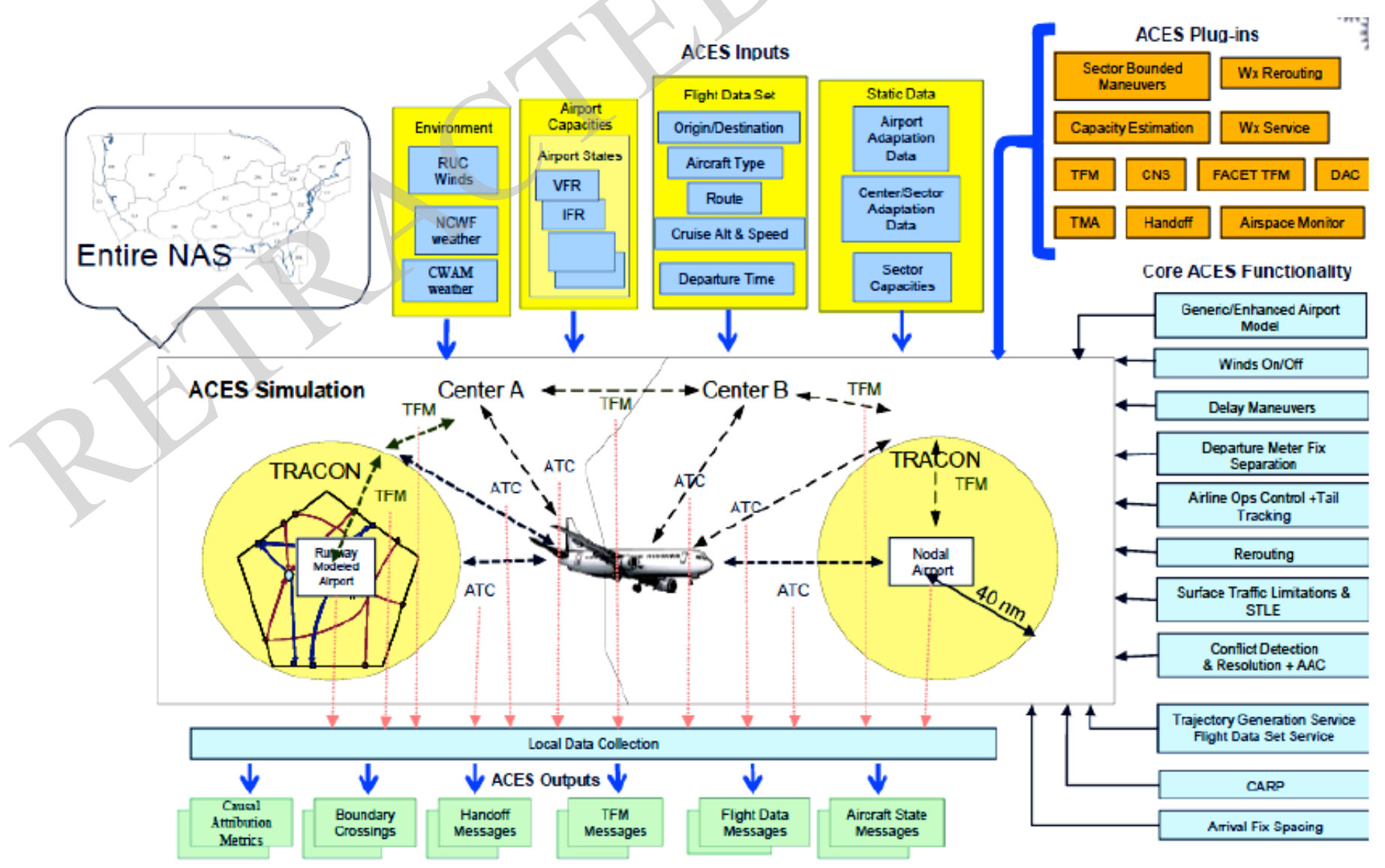

Fig. (6). The core functions and related plug-in. 
according with the feature of flight phase was established which combined dynamics with kinematics, and a mathematical method describing trajectory using state matrix and control matrix was purposed. Several satisfaction evaluation functions about noise impact, flight cost and air navigation were established based on fuzzy theory. Three heuristic search rules and dynamic neighborhood search method were presented to improve simulated annealing algorithm and case study and analysis was finished. Considering the lack of the emission index model developed by International Civil Aviation Organization, a amended model which could calculated accurately the e-mission of $\mathrm{HC}, \mathrm{CO}\}$ and $\mathrm{NO}\}$ was developed. A method similar to economy efficiency was used to analyze emission efficiency for flight procedure operation.

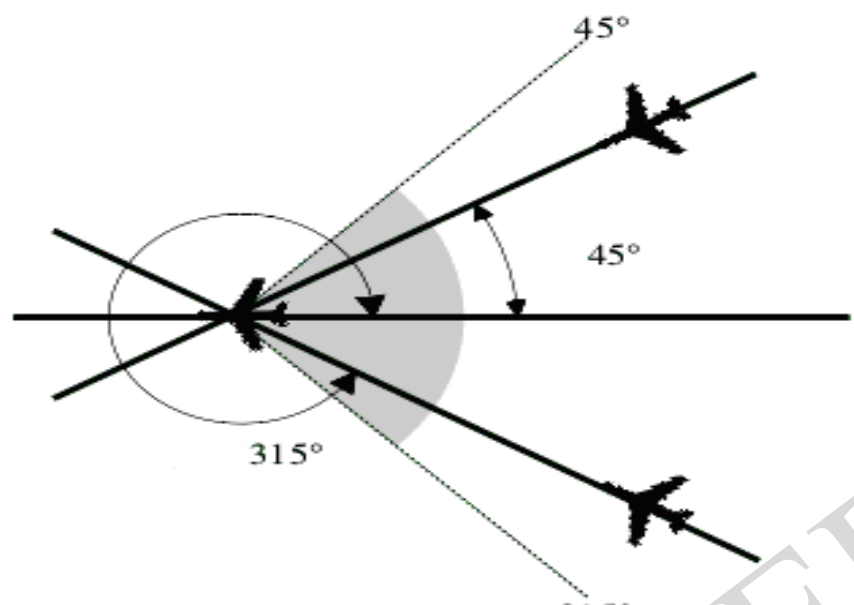

$315^{\circ}$

CSN9607 M $0978>980080$ ZUUU A320

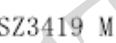
CS23419 M
$0978>980092$ ZUCK B747

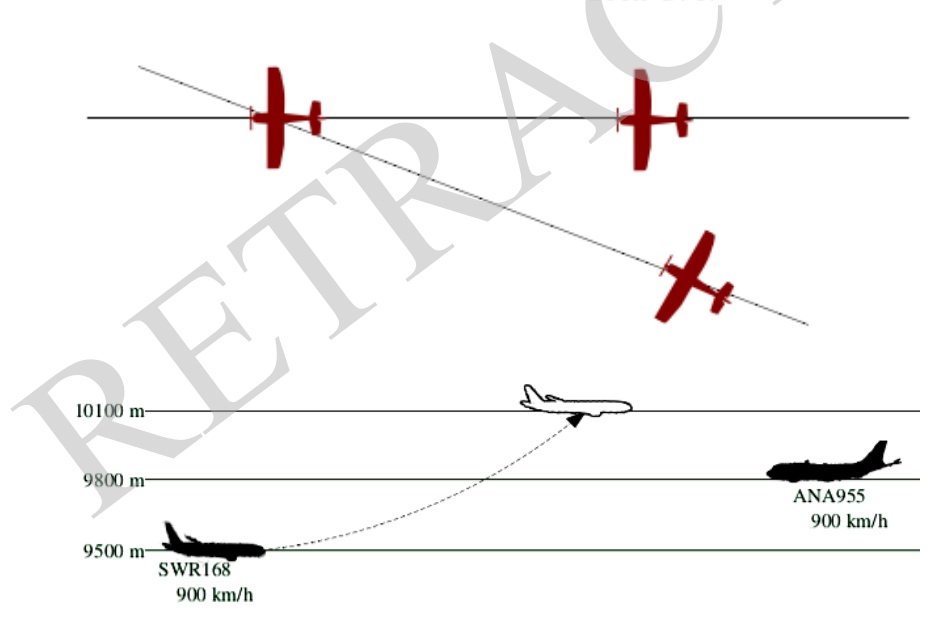

Fig. (7). Aircraft flight track.

Finally, the architecture of the flight procedure operation simulation and evaluation platform was studied and two subsystems include the flight operation simulation engine subsystem and the performance analysis subsystem were exposited on emphasis. A real terminal airspace was modeled as an example to design and carried out several synthetic simulation experiments.

The theories and methods for flight procedure operation's elevation were researched comprehensively and systematically, the assessment indexes and its measurement models are sensible and practical solutions, and a helpful simulation and analyzed platform has been developed correspondingly. The re-search achievements of the thesis completes the theories of flight procedure evaluation, but also cover the shortage of fundamental researches on simulation verification application platform in air traffic man-agreement, and will provide a technological support on the further and deep researches in analysis on the performance of air traffic system.

\section{SIMULATIONS}

Over the past decades, Object Oriented Technology connected with Systems simulation which makes a great advantage on different theories and methods, and been widely applied to the air traffic simulation. With the development of ATC simulation at home, real-time air traffic process simulation has become a hot focus and is in urgent need in air traffic simulation.

According to the condition of air traffic control, based on the real situation of Project Application, an airspace operation fuel economization model is put forward in the Air Traffic Process Simulation System, and by analyzing the result of flight operation simulation experiment, the feasibility of this model is proved (Fig. 8).

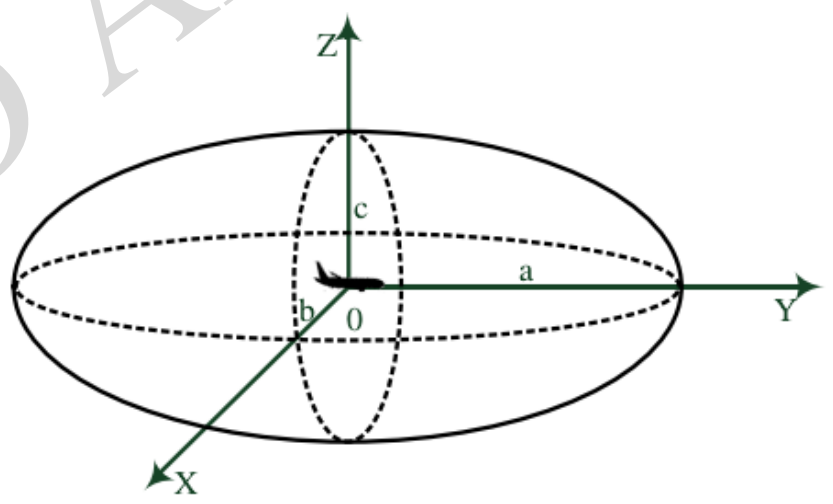

Fig. (8). Ellipsoidal reserve model diagram.

Air traffic network is constructed by the routes in airspace, and the air traffic flow operational system (ATFOS) is constructed by the movement of aircrafts flow flying in the air traffic network, air traffic flow management system (ATFMS) is built to guarantee ATFOS operation security, stability and efficient. The stability theory of ATFOS can provide scientific theory guidance for the establishment, safety and efficient management, and system maintenance of our country ATFMS. And also has a crucial significance on keeping ATFOS working in security, in stability and in order, making full use of facilities and airspace resources. The paper mainly research on the stability theory of air traffic flow operational system. As ATFOS is a large system, complex structures, many random factors and uncertainties and so on are the characteristics of ATFOS, we need analyze the system structure and its operation way before research on the stability. In this paper, foreign air traffic flow management systems are introduced, and then the organizational structure and operational structure of China's three level ATFMS are constructed based on multi-agent technology. Applying the multi-agent 
into the ATFM system can effectively enhance the system's adaptability and autonomy for dynamic environment, and reduce the complexity of design and construction of the system. The air traffic flow operation system states are sorted according to the stability degree; and the theory of transfer probability is used to study the state transfer of ATFMS in order to determine which pathway influences the system mostly and then effectively control the system (Fig. 9).

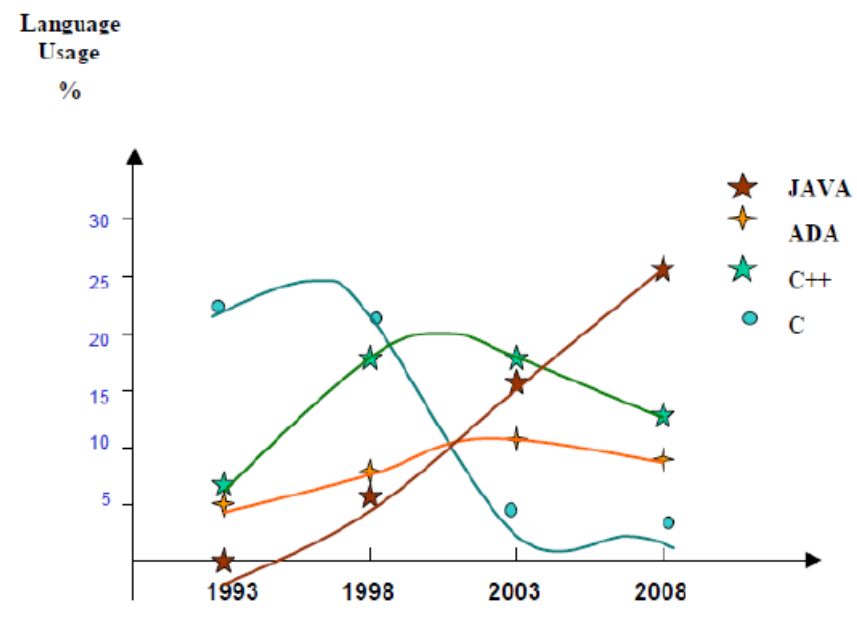

Fig. (9). Compared.

Introduce the research fundamental systematically. The operation concept of Collaborative Air Traffic Flow Management with its origin, content, research objectives and development are described first. After analyzing the detailed air transportation problem in practical, the four phases of the ATFM process in the CATFM Concept of Operations are explained. Finally, the paper presents the agent-based model with it communication method and the stochastic simulation modeling theory with detail.

Research has being done to multi-agent based collaborative flights planning problem. First, a multi-level collaborative flight planning procedure is established by means of collaboration between different air traffic management units and Airlines operations centers. Then, the agent-based modeling method is used to construct the core modeling and reasoning modules of air route traffic management units. Taking the summer and fall flight schedule of China in 2008 as data source, the potential planning problem of three main hub airports (ZBAA, ZSSS and ZGGG) in China are studied and the multi-objectives flight schedules are generated finally. See Eq. (1).

$S=V_{\text {current }} \times \frac{H_{\text {current }} \times H_{\text {accepet }}}{R_{\text {desecent }}}$

Research has being done to multi-agent based collaborative air routes management problem. First, an interactive collaborative air routes management procedure is established by means of collaboration between air routes traffic management units and Airlines operations centers. Then, the agent-based modeling method is used to construct the core operating and reasoning modules of air route traffic management units. After analyzing the radar data from Central and Southern China in2009, the ARENA tool is used here to conduct a discrete event simulation on the ZGGGZBAA route for validation finally. See Eq. (2).

$S=7 \times \frac{H_{\text {current }}-H_{\text {accepet }}}{300} \mathrm{~km}$

Research has being done to multi-agent based distributed collaborative aircraft operation. First, an adaptive collaborative aircraft performance procedure is established by means of collaboration between adjacent aircrafts.

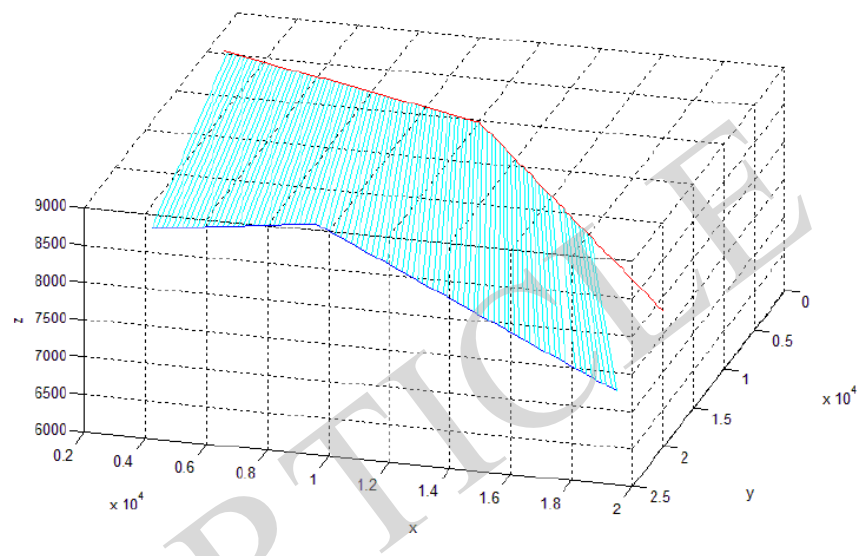

Fig. (10). The playback of the historical data.

Then, the agent-based modeling method is used to construct the core kinetic and reasoning modules of aircrafts by taking use of the point mass model and Proportionalderivative Controller. Taking the aircrafts operation performance parameters from the User Manual for the Base of Aircraft Data published by EUROCONTROL, a combined discrete continuous simulation of aircrafts flying in a self-separation corridor is conducted to check the performances of aircrafts finally. See Eq. (3).

$T_{2}=T_{1}+\frac{2 S_{0}}{V_{1}+V_{2}}=T+\frac{S_{0}+S_{0}}{V_{1}+V_{2}}$

In the end, the thesis summarizes the achievements of the collaborative air traffic flow management based upon multiagent modeling and simulation, discusses the limitation of this research and makes some suggestions for the future work. (Fig. 10) See Eq. (4).

$c=\sqrt{\left(a-v_{a} t\right)^{2}+\left(b-v_{b} t\right)^{2}-2\left(a-v_{a} t\right)\left(b-v_{b} t\right) \cos \theta}$

\section{CONCLUSION}

The researchers then developed a Multi-Agent framework JADE. Finally, the idea will be applied to the area of real-time operational control of Multi-Agent, for the proposed development of a prototype simulation system design, including functional module division, build a computer network communications.

First, the proposed strategy and deployment of aircraft types of activities, based controllers conflict and deployment algorithms; improved the severity of the conflict analysis algorithm; proposed aircraft Agent, programmable internal structure of ATC automation system controllers Agent and the Agent. Then, JADE been programmed, developed a prototype system simulation. 
Table 1. The program automatically generated to meet the conditions of flight plan.

\begin{tabular}{|c|c|c|c|c|c|c|c|c|}
\hline & (s) & (m) & $(\mathrm{km} / \mathrm{h})$ & & & & & \\
\hline 1 & 0 & 8300 & 886 & 1 & R1 & 10 & 10 & 6300 \\
\hline 2 & 287 & 8300 & 831 & 1 & $\mathrm{R} 1$ & 10 & 10 & 6300 \\
\hline 3 & 613 & 8300 & 894 & 2 & R1 & 10 & 10 & 6300 \\
\hline 4 & 897 & 8300 & 842 & 2 & R1 & 10 & 10 & 6300 \\
\hline 5 & 1213 & 8300 & 830 & 3 & $\mathrm{R} 1$ & 10 & 10 & 6300 \\
\hline 6 & 1478 & 8900 & 815 & 1 & R1 & 10 & 10 & 6300 \\
\hline 7 & 0 & 8300 & 813 & 1 & $\mathrm{R} 2$ & 20 & 120 & 6300 \\
\hline 8 & 309 & 8300 & 882 & 1 & $\mathrm{R} 2$ & 20 & 120 & 6300 \\
\hline 9 & 598 & 8300 & 868 & 2 & $\mathrm{R} 2$ & 20 & 120 & 6300 \\
\hline 10 & 907 & 8300 & 835 & 1 & $\mathrm{R} 2$ & 20 & 120 & 6300 \\
\hline 11 & 1301 & 8300 & 886 & 1 & $\mathrm{R} 2$ & 20 & 120 & 6300 \\
\hline 12 & 1507 & 8300 & 868 & 1 & $\mathrm{R} 2$ & 20 & 120 & 6300 \\
\hline
\end{tabular}

Finally, the prototype system was tested to verify, using three computers connected via a network. Use common control important scene-arriving aircraft convergence transfer terminal area. Test results show that three computers were running aircraft Agent, Agent ATC automation systems and controllers Agent, can be real-time interactive communications network, and more aircraft under the command of the two controllers Agent achieve a dynamic drop height, solve simple conflict can be handed two aircraft adjacent sectors.

\section{CONFLICT OF INTEREST}

The authors confirm that this article content has no conflict of interest.

\section{ACKNOWLEDGEMENTS}

Declared none.

\section{REFERENCES}

[1] Meyn, L.A. Preliminary Assessment of Future Operational Concepts Using the Airspace Concept Evaluation System. Reston, VA: American Institute of Aeronautics and Astronautics, AIAA, 2013

[2] DelaPeyronnie J.; Newcomb, P.H.; Morillo, V.; Trimech, F.; Nguyen, L.; Putyill, M. In Information Systems Transformation: Architecture-Driven Modernization Case Studies; William M. Ulrich, Philip Newcomb, Eds.; Elsevier Science B.V: Amsterdam, 2010, pp. 91-130.

[3] Portas, J.B. EATS: An Agent-based Air Traffic Simulator. Int. J. Comput. Sci. Info. Syst., 2008, 3(2), 36-50.

[4] Tumer, K. Improving Traffic Management with a Learning MultiAgent System. United States: The Software Revolution, Inc, 2010.

[5] Butler, R.; Maddalon, J.; Geser, A.; Munoz, C. Alfons Geser Cesar Munoz. Formal analysis of air traffic management systems: the case of conflict resolution and recovery. In: Proceedings of the 2003 Winter Simulation Conference 2003, pp. 906-914.

Yi, J.; Horowitz, R. Macroscopic Traffic Flow Stability for Adaptive Cruise Controlled (ACC) Vehicles. In: Proceedings of the 41 st IEEE Conference an Decision and Control Las Vegas Nevada USA, 2002, pp. 893-899.

[7] http://www. xavius. com/atasim. html

(c) Wei and Li; Licensee Bentham Open.

This is an open access article licensed under the terms of the Creative Commons Attribution Non-Commercial License (http://creativecommons.org/licenses/ by-nc/3.0/) which permits unrestricted, non-commercial use, distribution and reproduction in any medium, provided the work is properly cited. 